\title{
Endothelin 1: A Potential Prognostic Biomarker for Heart Failure with Preserved Ejection Fraction and Pulmonary Hypertension?
}

\author{
Xin He Hongli Guo Jing Xu \\ Department of Pharmacy, Children's Hospital of Nanjing Medical University, Nanjing, China
}

\section{Dear Editor,}

Heart failure (HF) is the major cause of mortality in patients with cardiovascular diseases. Patients with heart failure with preserved ejection fraction (HFpEF) who develop pulmonary hypertension $(\mathrm{PH})$ were shown to have increased short- and long-term mortality [1]. Better understanding of the pathophysiology behind this phenomenon and identifying prognostic markers are imperative to identify highrisk patients and improve outcomes. Recently, we read with great interest the article by Chowdhury et al. [2] published in Cardiology showing that endothelin 1 (ET1) is predictive of 1-year heart failure hospitalization in HFpEF patients, and elevated ET-1 levels were found to be associated with long-term mortality in HFpEF. This study highlights the role of ET-1 in developing $\mathrm{PH}$ in HFpEF patients and also explores the potential of ET-1 as a prognostic biomarker.

Other studies have been carried out on this topic. Meoli et al. [3] showed that ET-1 in patients with combined pre- and postcapillary $\mathrm{PH}(\mathrm{Cpc}-\mathrm{PH})$ was higher, compared to those without $\mathrm{PH}$ or with isolated postcapillary $\mathrm{PH}$. These findings implicate an elevated ET-1 as a marker of, and a potential contributor to, the development of $\mathrm{Cpc}-\mathrm{PH}$ in this population. Moreover, ET-1 is a 21 -amino acid peptide which is primarily produced in the pulmonary vascular endothelium and works on ET-A and ET-B receptors. On the contrary, the contribution of ET- 1 to abnormalities in endothelial function, vascular compliance, and $\mathrm{PH}$, implicates ET-1 in the pathophysiology of HFpEF. Therefore, ET-1 inhibition could have therapeutic applications in HFpEF. In support of this, Valero-Munoz et al. [4] demonstrated that dual ET-A/ET-B receptor inhibition improved HFpEF by abrogating adverse cardiac remodeling via antihypertrophic mechanisms and by reducing stiffness.

Collectively, the available evidence suggests a potential prognostic biomarker for HFpEF. Interestingly, ET-1 may serve as a potential prognostic marker for developing $\mathrm{PH}$ in HFpEF. Thus, further studies with larger sample sizes are required to validate that ET- 1 can potentially be used as a prognostic biomarker in the future. Early identification of HF patients with higher risk can lead to earlier intervention, which may potentially improve outcomes.

\section{Acknowledgment}

This work was funded by the Changzhou Siyao Foundation of Nanjing Pharmaceutical Association (2018YX003).

\section{Disclosure Statement}

There are no competing interests to declare. The authors have no conflicts of interest to disclose.

\section{References}

1 Cao TH, Quinn PA, Sandhu JK, Voors AA, Lang CC, Parry HM, et al. Identification of novel biomarkers in plasma for prediction of treatment response in patients with heart failure. Lancet. 2015 Feb;385 Suppl 1:S26.

2 Chowdhury MA, Moukarbel GV, Gupta R, Frank SM, Anderson AM, Liu LC, et al. Endothelin 1 Is Associated with Heart Failure Hospitalization and Long-Term Mortality in $\mathrm{Pa}$ tients with Heart Failure with Preserved Ejection Fraction and Pulmonary Hypertension. Cardiology. 2019;143(3-4):124-33.

3 Meoli DF, Su YR, Brittain EL, Robbins IM, Hemnes AR, Monahan K. The transpulmonary ratio of endothelin 1 is elevated in patients with preserved left ventricular ejection fraction and combined pre- and post-capillary pulmonary hypertension. Pulm Circ. 2018 Jan-Mar;8(1):2045893217745019.

4 Valero-Munoz M, Li S, Wilson RM, Boldbaatar B, Iglarz M, Sam F. Dual EndothelinA/Endothelin-B Receptor Blockade and Cardiac Remodeling in Heart Failure With Preserved Ejection Fraction. Circ Heart Fail. 2016 Nov;9(11):e003381.

karger@karger.com
www.karger.com/crd
Karger ${ }^{\prime /}$

Xin $\mathrm{He}$

Department of Pharmacy

Children's Hospital of Nanjing Medical University

72 Guangzhou Rd, Nanjing 210009 (China)

hexin19870422@163.com

Jing Xu

Department of Pharmacy

Children's Hospital of Nanjing Medical University

72 Guangzhou Rd, Nanjing 210009 (China)

njxujing2018@163.com 